\title{
A gyro-elastic device for cloaking of elastic waves in micro-structured materials
}

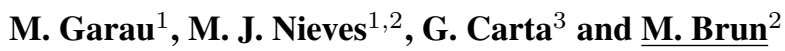 \\ ${ }^{1}$ Keele University, School of Computing and Mathematics, Keele, ST5 5BG, UK \\ ${ }^{2}$ University of Cagliari, Department of Mech., Chem. and Mat. Engineering, Cagliari, 09123, Italy \\ ${ }^{3}$ Liverpool John Moores University, Mech. Eng. and Mat. Research Centre, Liverpool, L3 3AF, UK. \\ mbrun@unica.it
}

\begin{abstract}
The design of a structured gyro-elastic system capable of being used as a cloaking device for a discrete medium is discussed. The efficiency of the gyro-elastic cloak, composed of springs connecting periodically placed masses, attached to gyroscopic spinners, is examined in the transient regime. An important effect encountered shows that the speed of the reconstructed field can be altered by tuning the gyroscopes.
\end{abstract}

\section{INTRODUCTION}

Structures capable of controlling waves for the purposes of cloaking are important in mitigating the catastrophic effects caused by the interaction of defects with waves. For continuous systems, the first designs of a cloaking device appeared in [1] for optics and [2] for electromagnetism. The notion of a continuum cloak for elastic waves was later developed in [3]. Lately, the design of gyro-elastic systems [4], i.e. structured media attached to arrays of gyroscopic spinners, has been paving new pathways in controlling vibrations in the time-harmonic regime, as demonstrated in [5, 6, 7]. In particular, the effective low-frequency properties of such systems were used in [4] to design an efficient cloaking and shielding device for a continuous elastic medium. More recently, transient models for a range of gyro-elastic systems were investigated and their applications to topological protection and cloaking were developed in [8]. Here, in the same spirit of $[4,8]$, we design a structured gyro-elastic cloaking device for a lattice, whose ability to cloak a defect is investigated in the transient regime.

\section{Model of A heXagonal gyro-elastic LatTice}

The design of the gyro-elastic cloak is based on the equations developed in [8] for an infinite homogeneous gyro-elastic lattice. The infinite lattice is as shown in Fig. 1(a), composed of springs of stiffness $c$ and length $L$ connecting a hexagonal array of nodes with mass $m$. The elementary cell of the lattice is described by the vectors $\mathbf{t}^{(j)}=L\left(3 / 2,(-1)^{j} \sqrt{3} / 2\right)^{\mathrm{T}}, j=1,2$, and contains two masses with displacements $\mathbf{u}_{k}^{(\mathbf{n})}, k=1,2$. Here $\mathbf{n}=\left(n_{1}, n_{2}\right)^{T}$ is a multi-index determining the elementary cell in the lattice. The junction points of the lattice are connected to a uniform array of gyroscopic spinners, whose motion is characterised by the generalised coordinates $\phi, \theta$ and $\psi$ representing its precession, nutation and spin. Each spinner has length $l$ and moments of inertia $I_{0}$ and

(a)

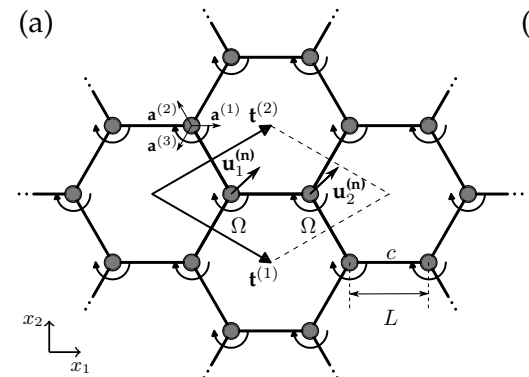

(b)

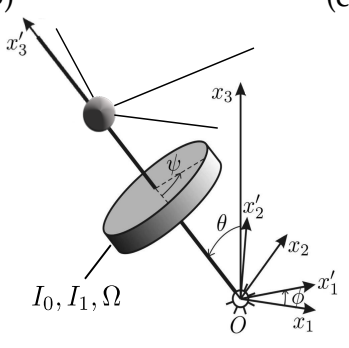

(c)

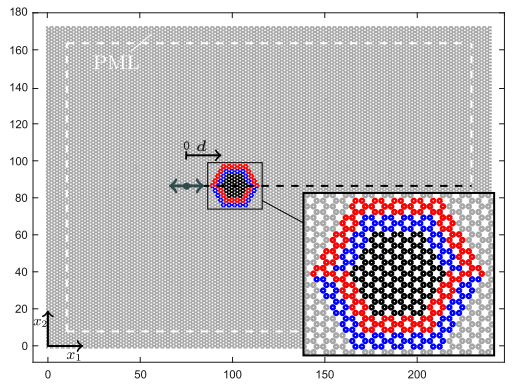

Fig. 1: (a) Infinite hexagonal lattice transversely supported by a uniform array of gyroscopic spinners shown in (b). (c) Computational geometry showing a hexagonal lattice with a hexagonal defect; in the magnification of the defect (represented by black circles), a cloak composed of a gyro-elastic medium (shown with red and blue circles) coats the defect. At the point $(75,50 \sqrt{3})$ a harmonic displacement is applied (represented by the black arrow). 
(a)

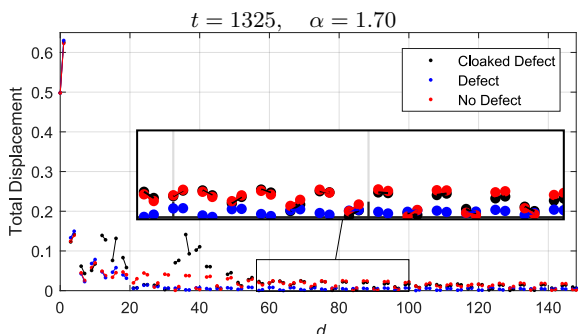

(c)

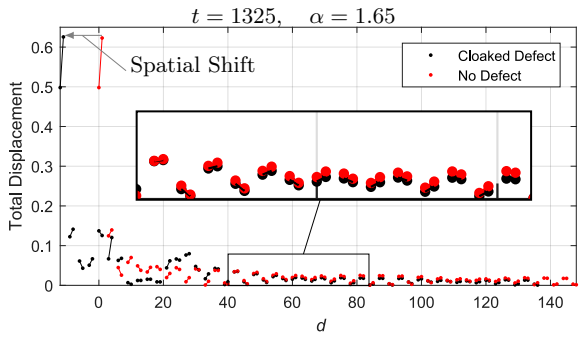

(b)

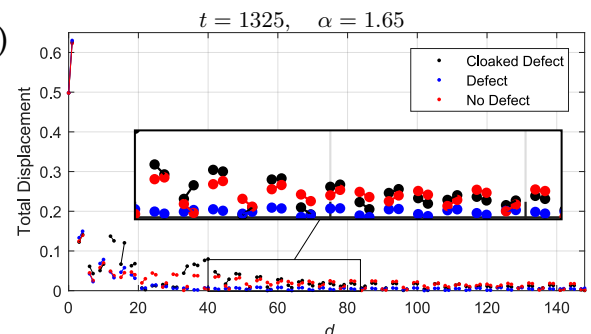

(d)

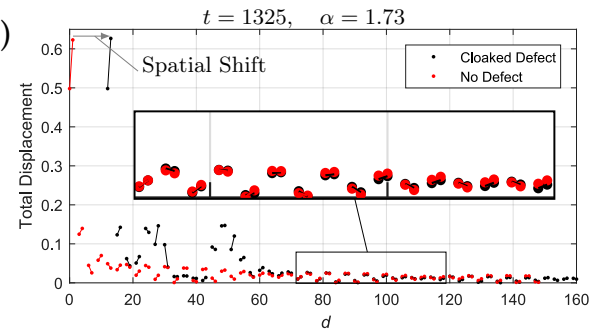

(e)

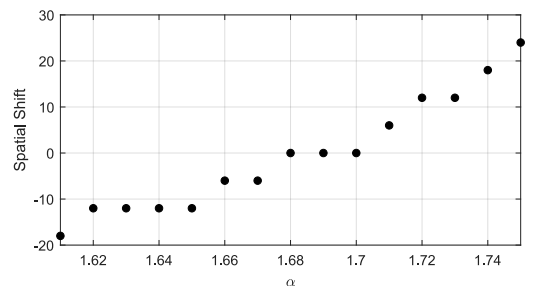

Fig. 2: Displacement magnitude of the nodal points intersecting the dashed line in Fig. 1(c) as a function of its local coordinate $d$, representing the distance from the source. Results are given at the normalised time $t=1325$ and in (a) $\alpha=1.70$ and (b) $\alpha=1.65$. In (c) $\alpha=1.65$ and (d) $\alpha=1.73$, the displacements for the case of the cloaked defect have been spatially shifted to the left and right, respectively. (e) The spatial shift as a function of $\alpha$.

$I_{1}$ about the $x_{1,2}^{\prime}$ and $x_{3}^{\prime}$ axes in Fig. 1(b), respectively. The masses are assumed to undergo small displacements, hence the resulting nutation $\theta$ of each spinner is small. In this case, the quantity $\dot{\psi}(t)+\dot{\phi}(t)$, i.e. sum of the spin and precession rates of the spinner, is constant. We denote by $\Omega=\dot{\psi}(0)+\dot{\phi}(0)$, the gyricity of the spinner and assume each spinner in the system possesses the same gyricity. We introduce the dimensionless time and gyricity through $\tilde{t}=t \sqrt{c l^{2} /\left(m l^{2}+I_{0}\right)}$ and $\tilde{\Omega}=\Omega / \sqrt{c l^{2} /\left(m l^{2}+I_{0}\right)}$, respectively. The dimensionless displacements of the nodes are $\tilde{\mathbf{u}}_{k}^{(\mathbf{n})}=\mathbf{u}_{k}^{(\mathbf{n})} / L$. In going forward we omit the symbol " ". In the transient regime the governing equations of the medium take the dimensionless form [8]:

$$
\ddot{\mathbf{u}}_{k}^{(\mathbf{n})}(t)=\sum_{j=1}^{3} \mathbf{a}^{(j)} \cdot\left\{\mathbf{u}_{3-k}^{\left(\mathbf{n}+(-1)^{k} \mathbf{p}_{j}\right)}(t)-\mathbf{u}_{k}^{(\mathbf{n})}(t)\right\} \mathbf{a}^{(j)}-\alpha \Omega \mathbf{R} \dot{\mathbf{u}}_{k}^{(\mathbf{n})}(t), \quad k=1,2,
$$

where $\mathbf{p}_{1}=(0,0)^{\mathrm{T}}, \mathbf{p}_{2}=(1,0)^{\mathrm{T}}, \mathbf{p}_{3}=(0,1)^{\mathrm{T}}$, the basis vectors $\mathbf{a}^{(1)}=(1,0)^{\mathrm{T}}$ and $\mathbf{a}^{(j)}=\left(-1 / 2,(-1)^{j} \sqrt{3} / 2\right)^{\mathrm{T}}$, for $j=2,3$. The last term in (1) represents the effective contribution of the gyroscopes to the motion of the system, which couple the in-plane motion of the masses, and

$$
\mathbf{R}=\left(\begin{array}{rr}
0 & 1 \\
-1 & 0
\end{array}\right), \quad \alpha=\frac{I_{1}}{\left(m l^{2}+I_{0}\right)} .
$$

\section{CloAking of ElAStiC WAVES IN THE MiCRO-STRUCTURED MEDiUM}

Consider a hexagonal elastic lattice containing a hexagonal defect as in Fig. 1(c). The lattice has normalised mass equal to one, while the stiffness of the links is 1 in the ambient medium and in the cloaking region (depicted in grey, blue and red) and 12 in the hexagonal defect (depicted in black). At the point $(75,50 \sqrt{3})$, an in-plane sinusoidal displacement is applied with amplitude $\mathbf{P}=(0.5,0)^{\mathrm{T}}$ and frequency $\omega=0.3$. The latter is chosen so that the ordinary hexagonal structure (shown in grey) is dynamically isotropic (see [8]). A numerical model has been implemented in Comsol Multiphysics 5.2a. The exterior of the domain is supplied with a layer of PMLs, 

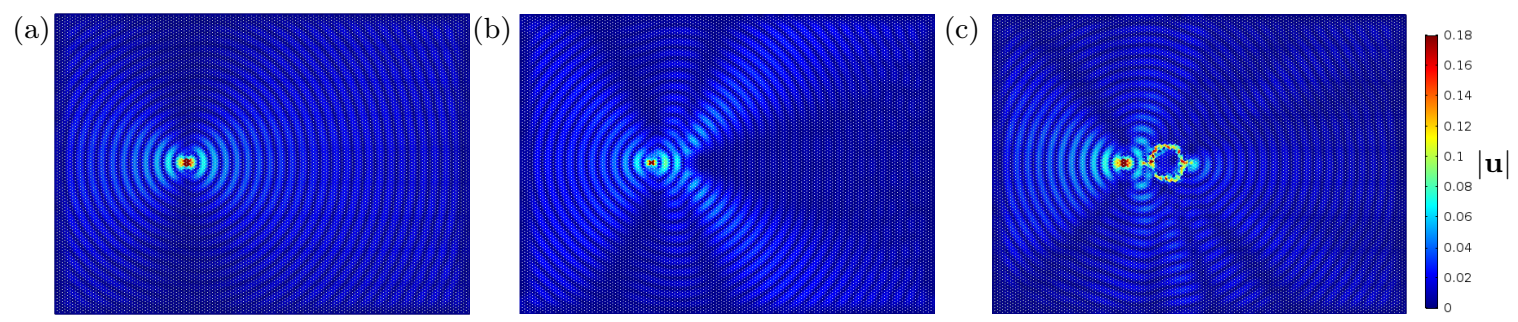

Fig. 3: The response of the (a) homogeneous lattice and the system with (b) the defect and (c) the cloaked defect with $\alpha=1.73$ taken at $t=1325$.

reducing the reflections of waves produced by the source or through scattering, thus simulating an infinite medium. Surrounding the defect is a heterogeneous gyro-elastic medium (the red and blue nodes in Fig. 1(c)). The red (blue) nodes possess gyricity $\Omega=-\omega(\Omega=\omega)$. The equations governing these nodes are analogous to (1).

Here, we analyse the effect of changing the parameter $\alpha$ on efficiency of the cloak in the transient regime. Fig. 2 shows the settled response along the horizontal dashed black line of Fig. 1(c) for the lattice with (i) no defect, (ii) the defect and (iii) the coated defect. Comparing cases (i) and (ii) in Figs. 2(a) and (b) it is clear that the defect creates a significant reduction of the wave field to the right of the defect. In this region, by comparing cases (i) and (iii), Fig. 2(a) shows a visibly excellent reconstruction of the field created by the cloak with $\alpha=1.7$.

The results for a cloak with $\alpha=1.65$ are shown Fig. 2(b), where it appears that the quality of the reconstruction of the field is not as good. Nevertheless, Fig. 2(c) demonstrates that by employing a spatial shift of 12 lattice units to the left, we retrieve an excellent match. For $\alpha=1.73$, Fig. 2(d) shows that the field matches the one in the homogeneous case for a spatial shift of 12 units to the right. Fig. 2(e) shows the spatial shift required for a good reconstruction of the field behind the defect as a function of $\alpha$. The spatial shift is a monotonically increasing function of $\alpha$. For $1.68 \leq \alpha \leq 1.7$ no spatial shift is required. For $\alpha$ below (above) this interval, a negative (positive) spatial shift is needed to demonstrate the capability of the cloak to reconstruct the field.

Finally, Fig. 3 shows the dynamic response of the system for the cases (i), (ii), and (iii). It can be concluded that apart from some clear preferential directions, Fig. 3 (c) shows a clear reconstruction of the field behind the defect.

\section{CONCLUSION}

We have illustrated the design of a discrete gyro-elastic cloak capable of hiding a defect within a vibrating structured system. The use of gyroscopic spinners in its design is crucial in mitigating the effects of interactions between a defect and elastic waves generated by a source applied near the defect. In particular, an important parameter characterising the type of spinner used has been identified and this provides the control of the apparent distance between the source and the external observer.

\section{REFERENCES}

[1] U. Leonhardt (2006): Optical conformal mapping, Science 312, (no. 5781), 1777-1780.

[2] J.B. Pendry, D. Schurig and D.R. Smith (2006): Controlling electromagnetic fields, Science, 312 (no. 5781), $1780-1782$.

[3] G.W. Milton, M. Briane and J.R. Willis (2006): On cloaking for elasticity and physical equations with a transformation invariant form, New J. Phys. 8, 248.

[4] M. Brun, I.S. Jones and A.B. Movchan (2012): Vortex-type elastic structured media and dynamic shielding, Proc. R. Soc. A 468, 3027-3046.

[5] G. Carta, M. Brun, A.B. Movchan, N.V. Movchan and I.S. Jones (2014): Dispersion properties of vortex-type monatomic lattices, Int. J. Solids Struct. 51, 2213-2225.

[6] G. Carta, I.S. Jones, N.V. Movchan, A.B. Movchan and M.J. Nieves (2017): “Deflecting elastic prism” and unidirectional localisation for waves in chiral elastic systems, Sci. Rep. 7, 26.

[7] M. Garau, G. Carta, M.J. Nieves, I.S. Jones, N.V. Movchan and A.B. Movchan (2018): Interfacial waveforms in chiral lattices with gyroscopic spinners, Proc. Roy. Soc. A. 474, 20180132.

[8] M. Garau, G. Carta, M.J. Nieves and M. Brun (2019): Transient analysis of a gyro-elastic structured medium: unidirectional waveforms and cloaking, Int. J. Eng. Sci. (in press).

\section{ACKNOWLEDGEMENT}

M.J.N and M.B. gratefully acknowledge the support of the EU H2020 grant MSCA-IF-2016-747334-CATFFLAP. G.C. would like to thank the EPSRC (UK) for its support through Programme Grant no. EP/L024926/1. 